\title{
NEW AREA EFFICIENT RESIDUE-TO-WEIGHTED NUMBER SYSTEM CONVERTERS
}

\author{
Jimson Mathew, D. Radhakrishnan and T. Srikanthan \\ Centre for High Performance Embedded Systems \\ Division of Computing Systems, SAS \\ Nanyang Technological University, Singapore 639798 \\ Email: asdrkrishnan@ntu.edu.sg
}

\begin{abstract}
Residue number system is popular in high performance arithmetic applications like digital signal processing because of its carry free nature, modularity and error correcting properties. But these opportunities are eclipsed by the high area and time requirements for reverse conversion. In this regard, we present two new techniques for residue-toweighted number system conversion. The first one is based on the popular Chinese Remainder Theorem. Here by evaluating the quotient the number is decoded. The second one deals with residue-to-mixed radix conversions. The arithmetic based technique replaces the conventional hardware intensive look up tables by simple adders. An OHR based high speed MRC is also presented. The mixed radix converters described are memoryless and are hardware efficient compared to conventional techniques.
\end{abstract}

\section{INTRODUCTION}

Residue number system (RNS) is popular in high performance arithmetic applications like Digital Signal Processing (DSP) systems because of its carry free nature, modular structure and error correcting properties [1,2]. It simplifies large number computation by decomposing them into a set of parallel independent computations on residues generated with respect to a convenient moduli set. Even though RNS is capable of performing high speed arithmetic, it was not popular in general computing because of the difficulties involved in division, magnitude comparisons, forward and reverse conversions between binary and residue numbers etc. Recent work in RNS provided a number of techniques to reduce the complexity of Residue-to-Binary Converters (RBC).

In RNS, an integer in the interval $[0, \mathrm{M})$ can be represented as a set of $n$ residues $\left(x_{1}, x_{2}, \ldots, x_{r}\right)$, with respect to a moduli set of $r$ pairwise relatively prime integers $\left(m_{1}\right.$, $\left.\mathrm{m}_{2}, \ldots, \mathrm{m}_{\mathrm{r}}\right) \quad$ where $\mathrm{x}_{\mathrm{i}}=\mathrm{X} \bmod \mathrm{m}_{\mathrm{i}}$ and $\mathrm{M}=\prod_{\mathrm{i}=1}^{\mathrm{r}} \mathrm{m}_{\mathrm{i}}$.

Arithmetic operations are computed by the formula:

$\left(\mathrm{x}_{1}, \mathrm{x}_{2}, \ldots, \mathrm{x}_{\mathrm{r}}\right) \otimes\left(\mathrm{y}_{1}, \mathrm{y}_{2}, \ldots, \mathrm{y}_{\mathrm{r}}\right)=\left(\mathrm{z}_{0}, \mathrm{z}_{2}, \ldots, \mathrm{z}_{\mathrm{r}}\right)$ where $\mathrm{z}_{\mathrm{i}}=\left|\mathrm{x}_{\mathrm{i}} \otimes \mathrm{y}_{\mathrm{i}}\right|_{\mathrm{m}_{\mathrm{i}}}$ and $\otimes$ denotes one of the operations of addition, subtraction or multiplication. Thus arithmetic operations on residues can be performed in parallel without any carry propagation among the residue digits. However, before performing any operations on residues, first the number has to be converted from binary-to-residue. The problem of translating a binary integer $X$ to the residue representation is called forward conversion. Getting back to the weighted representation of $\mathrm{X}$ from its residue representation is referred to as reverse conversion. The reverse conversion approach basically categorized into two groups. The first one is based on Chinese Remainder Theorem (CRT) and is given by: $X=\left|\sum_{i=1}^{r} a_{i} x_{i} \hat{M}_{i}\right|_{M}$ where $\hat{\mathrm{M}}_{\mathrm{i}}=\frac{\mathrm{M}}{\mathrm{m}_{\mathrm{i}}}$ and $\mathrm{a}_{\mathrm{i}}=\left|\frac{1}{\hat{\mathrm{M}}_{\mathrm{i}}}\right|_{\mathrm{m}_{\mathrm{i}}}$.

Various CRT implementations based on classical formulation or its adaptations have been proposed [3 - 5]. In [3] the contribution of each bit of the $r$ residues is added using a linear array of adders that emulates a binary tree in time. For an $n$ bit modulus, a total of r.n/2 adders are used for the whole converter. The same set of adders are repeatedly used until the final residue is generated. The main drawbacks of this technique include considerable non-local communication, large area overhead and high conversion time besides non trivial control and clocking. In [4] groups of bits are considered (as against single bits in [3]) and their contributions are added using a dedicated binary tree of adders. For moduli sets where the number of moduli scales with dynamic range this solution does not provide any results better than [3]. In [5] the authors use another form of CRT, as given by the expression: $X=\sum_{i=1}^{r} \beta_{i} \hat{M}_{i}$, where $\beta_{i}=\left|\frac{X_{i}}{\hat{M}_{i}}\right|_{m_{i}}$, and suggests a method to compute $\beta_{i} \hat{M}_{i}$. The evaluation $\beta_{i} \hat{M}_{i}$ is done by splitting $\hat{M}_{i}$ into groups of $b$ bits each and multiplying $\beta_{\mathrm{i}}$ by each of the high radix bits while avoiding carry propagation across these groups. This results in a carry propagation only in the last step, when the two radix $\mathrm{B}$ digits are added to reduce it to a number correct to $\bmod M$. This provides a highly efficient reverse conversion architecture based on $\mathrm{AT}^{2}$ minimization using both conventional and modular multipliers. In [10] the authors use the same CRT equation that was used in [5], but use a different technique for computing $\beta_{\mathrm{i}} \hat{M}_{\mathrm{i}}$. This is done by the Booth recording of $\hat{\mathrm{M}}_{\mathrm{i}}$ (a shift and add technique). The main drawback is due to the two carry propagation stages following the Carry Save Adder (CSA) tree. In another approach [9,11], first the value $\left|\mathrm{a}_{\mathrm{i}} \mathrm{x}_{\mathrm{i}} \hat{\mathrm{M}}_{\mathrm{i}}\right|_{\mathrm{M}}$ is precomputed and then stored in a table of width $\left\lceil\log _{2}(M-1)\right\rceil$. Now based on input value $x_{i}$ the appropriate entries are selected from the tables and accumulated and then a modulo correction is performed. The value of $\mathrm{X}$ is now given by: $X=\left.\left.\left|\sum_{i=1}^{r}\right| a_{i} X_{i} \hat{M}\right|_{M}\right|_{M}$. 
A second approach uses mixed radix conversion (MRC) and is given by the following equation

$$
\begin{aligned}
& \mathrm{X}=\mathrm{A}_{\mathrm{r}} \prod_{\mathrm{i}=1}^{\mathrm{r}-1} \mathrm{~m}_{\mathrm{i}}+\ldots+\mathrm{A}_{3} \mathrm{~m}_{2} \mathrm{~m}_{1}+\mathrm{A}_{2} \mathrm{~m}_{1}+\mathrm{A}_{1} \text { where } \\
& \mathrm{A}_{\mathrm{i}} \in\left[0, \mathrm{~m}_{\mathrm{i}}\right) \text { and } \\
& \mathrm{A}_{\mathrm{i}}=\| \frac{1}{\mathrm{~m}_{1} \mathrm{~m}_{2} \cdots \mathrm{m}_{\mathrm{i}-1}}\left|\left(\mathrm{x}_{\mathrm{i}}-\left(\mathrm{A}_{\mathrm{i}-1} \mathrm{~m}_{\mathrm{i}-2} \ldots \mathrm{m}_{1}+\ldots+\mathrm{A}_{1}\right)\right)\right|_{\mathrm{m}_{\mathrm{i}}}
\end{aligned}
$$

It is clear from the expressions above that MRC is an inherently serial approach. Also, the conversion procedure requires a total of $\mathrm{r}(\mathrm{r}-1) / 2$ modulo multiplications and modulo subtractions. The traditional techniques use a look up table approach by precomputing the values and storing them in a table. The block schematic representation is shown in Figure 1. These stored values are accessed in a serial fashion [12]. The main drawback of this method is that it is hardware intensive and as bits per modulus increases the hardware grows exponentially.

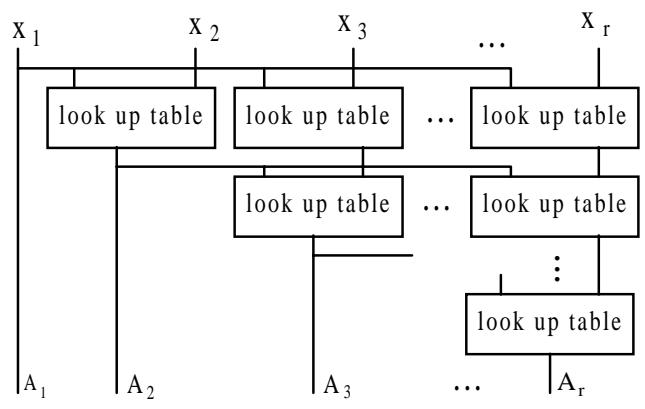

Fig. 1: Look up Table Based MRC

In this paper an efficient method for reducing the hardware implementation using CRT and MRC techniques are presented. Section 2 introduces the proposed converter followed by a performance evaluation. In Section 3 the new memory less residue-to-mixed radix converters are introduced and a comparison is made with respect to the traditional look up table approach. Finally Section 4 offers conclusions.

\section{DECODING USING QUOTIENT EVALUATION}

One of the major problems with the above CRT implementation is its hardware complexity. To reduce the hardware complexity we propose the following modification. This is done by evaluating the quotient alone so that it always uses less hardware. The only requirement we impose is that the moduli set contains the modulus $2^{\mathrm{n}}$, where $\mathrm{n}$ is an integer. The reduction in the size of the ROM table is based on the following theorem.

THEOREM 1: In an RNS with moduli set $\left(\mathrm{m}_{1}, \mathrm{~m}_{2}, \ldots, \mathrm{m}_{\mathrm{r}}\right)$, where $m_{r}=2^{n}$, if an integer $X$ is represented in residue form $\left\lfloor\frac{\mathrm{X}}{\mathrm{m}_{\mathrm{r}}}\right\rfloor=\left|\frac{1}{\mathrm{~m}_{\mathrm{r}}} \sum_{\mathrm{i}=1}^{\mathrm{r}-1} \mathrm{a}_{\mathrm{i}} \mathrm{x}_{\mathrm{i}} \hat{\mathrm{M}}_{\mathrm{i}}+\left\lfloor\frac{1}{\mathrm{~m}_{\mathrm{r}}} \mathrm{a}_{\mathrm{r}} \mathrm{x}_{\mathrm{r}} \hat{\mathrm{M}}_{\mathrm{r}}\right\rfloor\right|_{\hat{\mathrm{M}}_{\mathrm{r}}}$ where $\hat{M}_{i}=\frac{M}{m_{i}}$, and $M=\prod_{i=1}^{r} m_{i}$.

Because of space constraints the proof is omitted here.

In general, $\mathrm{X}$ can always be represented as: $\mathrm{X}=\mathrm{km}_{\mathrm{r}}+\mathrm{X}_{\mathrm{r}}$, where $m_{r}=2^{n}$. Then the integer $X$ can be computed by shifting $\mathrm{k}$ by $\mathrm{n}$ bit positions to the left and filling the lowest $\mathrm{n}$ bits with $\mathrm{x}_{\mathrm{r}}$. From Theorem 1 this amounts to calculating the value of $\mid \frac{1}{\mathrm{~m}_{\mathrm{r}}} \sum_{\mathrm{i}=1}^{\mathrm{r}-1} \mathrm{a}_{\mathrm{i}} \mathrm{x}_{\mathrm{i}} \hat{\mathrm{M}}_{\mathrm{i}}+\left\lfloor\frac{1}{\mathrm{~m}_{\mathrm{r}}} \mathrm{a}_{\mathrm{r}} \mathrm{x}_{\mathrm{r}} \hat{\mathrm{M}}_{\mathrm{r}} \|_{\hat{\mathrm{M}}_{\mathrm{r}}}, \quad\right.$ that represents $\mathrm{k}$. If we use this technique shown in Figure 2 to implement the reverse converter, the ROM's used to generate the partial sum $\left|\frac{1}{m_{r}} a_{i} X_{i} \hat{M}_{i}\right|_{\hat{M}_{r}}$ need only be $\left\lceil\log _{2}(\mathrm{M}-1)\right\rceil-\mathrm{n}$ bits wide as compared to $\left\lceil\log _{2}(\mathrm{M}-1)\right\rceil$ bits used in earlier approaches. Hence there is always a reduction of $\mathrm{n}$ bits/word in each of the ROMs. Once $\mathrm{k}$ is calculated, $\mathrm{X}$ is obtained by concatenating $\mathrm{k}$ and $\mathrm{x}_{\mathrm{r}}$.

\subsection{Performance Evaluation}

Figure 2 shows the general scheme of the modified residue-to-binary converter. Functionally the proposed architecture is similar to the converter given in [11]. In our approach the width of the ROMs which perform the look up operation has been reduced by $\mathrm{n}$ bits. This is achieved by storing the contents with respect to modulus $\hat{\mathbf{M}}_{\mathrm{i}}$ instead of M. This amounts to an overall reduction of $2^{n} \times n \times r$ bits of ROM for the entire design. Furthermore this reduces the size of the CSA tree and the accompanying CSA, CPA and MUX stages in comparison to the design given in [11]. Table 1 shows the comparison results. For example if we compare

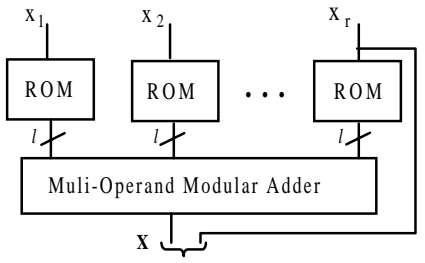

Fig. 2: Proposed Residue-to-Binary Converter

in terms of transistor count there is nearly $20 \%$ reduction in ROM requirement and $25 \%$ reduction in full adders. And a similar reduction is seen in the MSB converter and output MUX. There is $20 \%$ delay reduction assuming CPAs.

The following notations from [10] are assumed in Table 1: $t_{\mathrm{FA}}$ - the delay of a Full Adder; $t_{\mathrm{MUX}}$ - Delay of a twoinput multiplexer; $t_{\text {con(p) }}$ the delay of an a-input 1 -output converter; $\mathrm{t}_{\mathrm{CPA}(\mathrm{p})}$ - the delay of a $\mathrm{p}$ bit carry propagate adder; $\mathrm{t}_{\mathrm{CLA}(\mathrm{p})^{-}}$the delay of a p-bit CLA; $\theta(\mathrm{N})$-the minimum number of levels in the CSA tree with $\mathrm{N}$ operands.

\section{MEMORYLESS MRC}

Mixed radix converters proposed so far are based on a look up tables [12]. In this section we propose a new approach whereby all look up tables in previous implementations are replaced by arithmetic units. The basic idea is that instead of precomputing the values corresponding to the coefficients, we compute the values in real time. The 
method can be explained as follows. In a typical look up table implementation the function of each block is to perform a modulo subtraction and a modulo multiplication by a constant.

In general, if two inputs to a block are labeled as $\mathrm{X}$ and $\mathrm{Y}$ each $\mathrm{n}$ bits wide, then the outputs of the block are given by: $\mathrm{z}=|(\mathrm{X}-\mathrm{Y}) \mathrm{k}|_{\mathrm{m}}$

where $\mathrm{k}$ is an integer constant, and $\mathrm{X}$ and $\mathrm{Y}$ can be expressed as $\mathrm{X}=2^{\mathrm{n}-1} \mathrm{x}_{\mathrm{n}-1}+2^{\mathrm{n}-2} \mathrm{x}_{\mathrm{n}-2}+\cdots+\mathrm{x}_{0}$, and

$\mathrm{Y}=2^{\mathrm{n}-1} \mathrm{y}_{\mathrm{n}-1}+2^{\mathrm{n}-2} \mathrm{y}_{\mathrm{n}-2}+\cdots+\mathrm{y}_{0}$. Substituting in Equation 4.1 we have,

$z=\left|\begin{array}{l}\left(\left(2^{n-1} x_{n-1}+2^{n-2} x_{n-2}+\cdots+x_{0}\right)-\right. \\ \left.\left(2^{n-1} y n-1+2^{n-2} y_{n-2}+\cdots+y_{0}\right)\right) k\end{array}\right|_{m}$

Simplifying

$$
\begin{aligned}
\mathrm{z} & =\left|\begin{array}{l}
\left|2^{\mathrm{n}-1} \mathrm{k}\right|_{\mathrm{m}} \mathrm{x}_{\mathrm{n}-1}+\left|2^{\mathrm{n}-2} \mathrm{k}\right|_{\mathrm{m}} \mathrm{x}_{\mathrm{n}-2}+\cdots+|\mathrm{k}|_{\mathrm{m}} \mathrm{x}_{0}- \\
\left(\left|2^{\mathrm{n}-1} \mathrm{k}\right|_{\mathrm{m}} \mathrm{y}_{\mathrm{n}-1}+\left|2^{\mathrm{n}-2} \mathrm{k}\right|_{\mathrm{m}} \mathrm{y}_{\mathrm{n}-2}+\cdots+|\mathrm{k}|_{\mathrm{m}} \mathrm{y}_{0}\right)
\end{array}\right|_{\mathrm{m}} \\
& =\left|\begin{array}{l}
\left(\mathrm{v}_{\mathrm{n}-1} \mathrm{x}_{\mathrm{n}-1}+\mathrm{v}_{\mathrm{n}-2} \mathrm{x}_{\mathrm{n}-2}+\cdots+\mathrm{v}_{0} \mathrm{x}_{0}\right)- \\
\left(\mathrm{v}_{\mathrm{n}-1} \mathrm{y}_{\mathrm{n}-1}+\mathrm{v}_{\mathrm{n}-2} \mathrm{y}_{\mathrm{n}-2}+\cdots+\mathrm{v}_{0} \mathrm{y}_{0}\right)
\end{array}\right|_{\mathrm{m}} \\
& =\mid \begin{array}{l}
\left(\mathrm{v}_{\mathrm{n}-1}\left(\mathrm{x}_{\mathrm{n}-1}-\mathrm{y}_{\mathrm{n}-1}\right)+\mathrm{v}_{\mathrm{n}-2}\left(\mathrm{x}_{\mathrm{n}-2}-\mathrm{y}_{\mathrm{n}-2}\right)\right. \\
\left.+\cdots+\mathrm{v}_{0}\left(\mathrm{x}_{0}-\mathrm{y}_{0}\right)\right)
\end{array}
\end{aligned}
$$

where $\mathrm{v}_{\mathrm{i}} \in[0, \mathrm{~m})$ and $\mathrm{z}$ is the output of a block which implements Equation 4.1.

The term $x_{i}-y_{i}$ may take values 1,0 or -1 . Hence the modulo subtraction and modulo multiplication by a constant in Equation 4.1 have been reduced to the addition/ subtraction of the numbers $\mathrm{v}_{\mathrm{i}}$. An architecture to implement the mixed radix converter based on this principle can be implemented using adders and simple combinational logic. The schematic representation is shown in Figure 3. To compare the hardware requirement assume that we have an RNS with 5 bits per modulus. In this case each of the inputs is five bits and the output is also five bits, so the look up table size required is $2^{10} \times 5$ bits. That is, 640 bytes of ROM per table,

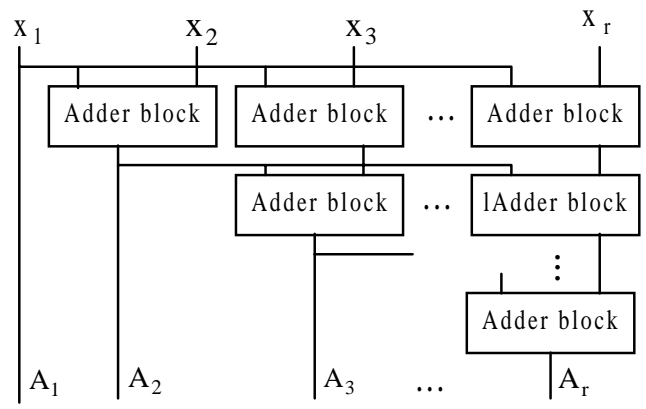

Fig. 3: Adder Based MRC

thus requiring a total of 3840 bytes. This needs a total transistor count of 6597 to implement one look up table. The number of transistors required to implement a single adder block in the method described is 1040 . This is considerably less than the number of transistors used (6597) in the earlier approach. Table 2 shows the requirement for various moduli, having different bit lengths. We can see that the hardware requirement grows exponentially for a look up table approach, whereas it is more or less linear with respect to the number of bits per moduli in our adder based approach.

An alternative method of eliminating the look up tables in MRC is by making use of the property of One-hot Residue Number system (OHR) described in [14]. With this one-hot coded representation of the residue digits, addition can be performed by cyclic shifts (rotations). One of the operands is rotated by an amount equal to the other operand. The rotation can be performed by barrel shifters. These circuits compute all possible rotations in parallel and pass when required the appropriate one to the output. The barrel shifters can be built using pass transistors or transmission gates. A subtractor is implemented in the same way, except that the subtrahend input bus is permuted to generate the additive inverse (modulo $\mathrm{m}_{\mathrm{i}}$ ) of its operand. Each block of Figure 1 performs a modulo subtraction and modulo multiplication by a constant. These blocks can be replaced by OHR cell, which is shown in Figure 4. Therefore we can use an OHR subtractor here and multiplication by a constant modulo $\mathrm{m}_{\mathrm{i}}$ is done by wire transposition. This approach is better when conversion speed is important.

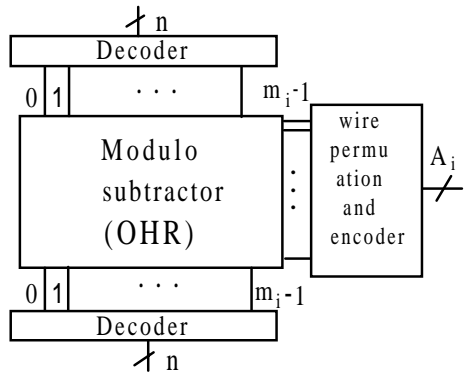

Fig. 4: Modulo subtraction and muliplication using OHR

Table 2 shows the hardware requirement in terms of transistors for different moduli, having different bit lengths. We can see that the hardware requirement grows exponentially for a look up table approach, whereas it is more or less linear with respect to the number of bits per moduli in adder based approach. The hardware growth is at a quadratic rate with OHR blocks.

Table 2: Comparison of various MRC Implementations

\begin{tabular}{|l|l|l|l|l|}
\hline $\begin{array}{l}\text { Sl. } \\
\text { No }\end{array}$ & $\begin{array}{l}\text { Bits per } \\
\text { mod. }\end{array}$ & $\begin{array}{l}\text { Look up } \\
\text { table }\end{array}$ & $\begin{array}{l}\text { Adder } \\
\text { based }\end{array}$ & $\begin{array}{l}\text { OHR } \\
\text { Based }\end{array}$ \\
\hline $\mathbf{1}$ & $\mathbf{3}$ & $\mathbf{3 7 1}$ & $\mathbf{4 8 6}$ & $\mathbf{2 5 6}$ \\
2 & 4 & $\mathbf{1 3 3 2}$ & $\mathbf{8 9 6}$ & $\mathbf{8 3 2}$ \\
3 & $\mathbf{5}$ & $\mathbf{6 5 9 7}$ & $\mathbf{1 0 4 0}$ & $\mathbf{2 5 6 0}$ \\
4 & 6 & $\mathbf{2 8 4 9 8}$ & $\mathbf{2 1 1 2}$ & $\mathbf{6 4 0 0}$ \\
$\mathbf{5}$ & 7 & $\mathbf{1 2 4 6 7 9}$ & $\mathbf{2 9 1 2}$ & $\mathbf{2 5 6 0 0}$ \\
6 & $\mathbf{8}$ & $\mathbf{5 4 9 1 2 8}$ & $\mathbf{3 5 8 4}$ & $\mathbf{8 7 0 4 0}$ \\
7 & 9 & $\mathbf{2 4 1 9 7 2 1}$ & $\mathbf{4 5 3 6}$ & $\mathbf{3 1 1 2 9 6}$ \\
8 & 10 & $\mathbf{1 0 5 6 8 7 1 4}$ & $\mathbf{5 3 6 0}$ & $\mathbf{1 1 5 9 1 6 8}$ \\
\hline
\end{tabular}

\section{CONCLUSIONS}

In this paper efficient residue-to-weighted number system converters for RNS are presented. A new technique has been introduced to reduce the complexity of the converter design 
by evaluating the quotient alone and the number $\mathrm{X}$ is deduced from the quotient. The second approach is for residue to mixed radix conversion, that replaces the look up table approach by simple adders and OHR cells. The OHR based conversion is preferred in places where speed of conversion is important. The total hardware requirement has been shown to be less than that used in traditional designs.

\section{REFERENCES}

[1] N.W. Szabo and R.I. Tanaka, Residue Number Arithmetic and its Application to Computer Technology. New York: McGraw Hill, 1967.

[2] M.A. Soderstrand, W.K. Jenkins, G.A. Jullien, and F.J. Taylor, Residue Number System Arithmetic: Modern Applications in Digital Signal Processing. New York: IEEE Press, 1986.

[3] G. Alia and E. Martinelli, "A VLSI algorithm for direct and reverse conversion from weighted binary number system to residue number system," IEEE Trans. CAS, vol. 31, pp. 1033-1039, Dec. 1984.

[4] K.M. Elleithy and M.A. Bayoumi, "Fast and flexible architectures for RNS arithmetic decoding," IEEE Trans. CAS-II, vol. 39, no. 4, pp. 226-235, Apr. 1992,

[5] F. Barsi and M.C. Pinotti, "A fully parallel algorithm for residue-to-binary conversion," Inform. Proc. Letters, vol. 50, pp. 403-407, 1994.

[6] G. Bi and E.V. Jones, "Fast conversion between binary and residue numbers," Electronic Letters, vol. 24, no. 19, pp. 195-1197, Sept. 1988.
[7] K.M. Ibrahim and Saloum, "An Efficient Residue-tobinary Converter Design," IEEE Trans. CAS, vol. 35, no. 9, pp. 1156-1158, Sept. 1988.

[8] S.J. Piestrak, "Design of Residue Generators and Multioperand Modular Adders Using Carry Save Adders," IEEE Trans. Comput., vol. 423, no. 1, pp. 6877, Jan. 1994.

[9] B. Parhami and C.Y. Huang, "Optimal look up Schemes for VLSI implementation of input/output conversions and other residue number operations," in VLSI Signal Processing VII, J. Rabaey, P. M. Chau and Eldon, eds, New York: IEEE Press, 1994.

[10] T. Srikanthan, M. Bhardwaj and C.T. Clarke, "Areatime-efficient VLSI residue-to-binary converters," IEE Proc. Comput. Digit. Tech., vol. 145, no. 3, pp. 229-235, May 1998.

[11] S.J. Piestrak, "Design of High-speed Residue-to-Binary Number System Converter Based on Chinese Remainder Theorem," Proc. IEEE Intl. Conf. on Computer Design: VLSI in Computers \& Processors, 1994, pp. 508-511.

[12] D.F. Miller, W. S. McCormick, "An Arithmetic Free Parallel Mixed-Radix Conversion Algorithm," IEEE Trans. Circuits Syst.-II: Analogue and Digital Signal processing, vol. 45, no. 1, pp. 158-162, Jan. 1998.

[13] C.H. Huang," A fully parallel mixed radix conversion algorithm for RNS applications,"IEEE Trans. Comput., vol. 32, pp. 398-402, Apr. 1983.

[14] W. A. Chren, "One- Hot Residue Coding for Low Delay-Power Product CMOS Design," IEEE Trans. Circuits Syst.-II: Analogue and Digital Signal Processing, vol. 45, no. 3, pp. 303-313, Mar. 1998

Table 1: Quotient Evaluation Vs. Chinese Remainder Theorem

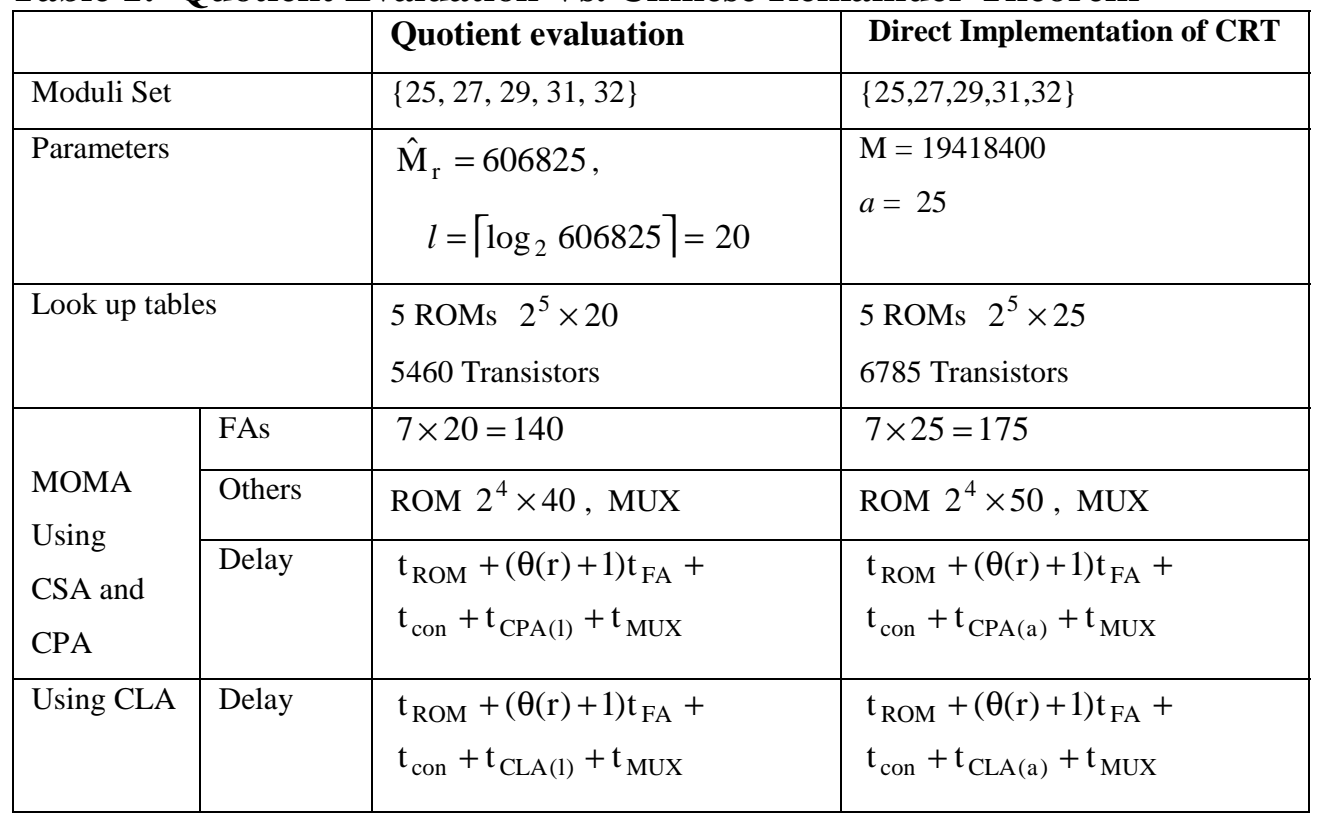

